\title{
Positron-induced luminescence
}

\author{
E. V. Stenson, ${ }^{1, *}$ U. Hergenhahn, ${ }^{1,2}$ M. R. Stoneking,,${ }^{1,3}$ and T. Sunn Pedersen ${ }^{1,4}$ \\ ${ }^{1}$ Max Planck Institute for Plasma Physics, 17491 Greifswald and 85748 Garching, Germany \\ ${ }^{2}$ Leibniz Institute of Surface Modification, 04318 Leipzig, Germany \\ ${ }^{3}$ Department of Physics, Lawrence University, Appleton, WI, 54911, U.S.A. \\ ${ }^{4}$ University of Greifswald, 17489 Greifswald, Germany
}

(Dated: February 12, 2018)

\begin{abstract}
We report on the observation that low-energy positrons incident on a phosphor screen produce significantly more luminescence than electrons do. For two different wide-bandgap semiconductor phosphors ( $\mathrm{ZnS}: \mathrm{Ag}$ and $\mathrm{ZnO}: \mathrm{Zn}$ ), we compared the luminescent response to a positron beam with the response to an electron beam. For both phosphors, the positron response is significantly brighter than the electron response, by a factor that depends strongly on incident energy $(0-5 \mathrm{keV})$. Positrons with just a few tens of eV of energy (for $\mathrm{ZnS}: \mathrm{Ag}$ ) or less (for $\mathrm{ZnO}: \mathrm{Zn}$ ) produce as much luminescence as is produced by electrons with several $\mathrm{keV}$. We attribute this effect to valence band holes and excited electrons produced by positron annihilation and subsequent Auger processes. These results demonstrate a valuable approach for addressing longstanding questions about luminescent materials.
\end{abstract}

Cathodoluminescent materials, in addition to being widespread in consumer devices, are important scientific tools for diagnosing charged particle beams, short wavelength photons, and non-neutral plasmas. Despite decades of use, however, some important aspects of the physics of phosphors remain topics of debate and investigation [1-3]. Depending on the material, this may include the structure of luminescence centers $[3,4]$, the excitation and relaxation pathways $[1,5]$, and/or the origin of the dead voltage $[2,6]$. (For most phosphors, no luminescence is observed when incident electrons' kinetic energy is below some threshold, which is usually orders of magnitude larger than the material's band gap. The transition to the linear regime, in which an increase in electron beam energy produces a proportional increase in the number of photons produced, may not occur until the energy exceeds several keV.) Yet another question involving phosphors is a practical one; although they are routinely used for diagnosing both matter and antimatter systems [7], comparisons between the two seem to be scarce. Quantitative studies of positron luminescence with higher-energy beams $(\geq 30 \mathrm{keV})$ have been performed on two different materials $\left(\mathrm{C}_{14} \mathrm{H}_{10}\right.$ and $(\mathrm{ZnS}$, $\mathrm{CdS}): \mathrm{Ag}$ ), in which no significant differences from the electron response were observed $[8,9]$, as has a qualitative study at lower energies in a polymer solution [10]. However, we were unable to find in the electronically searchable literature a quantitative comparison of the luminescence produced by low-energy $(0-5 \mathrm{keV})$ positrons and electrons incident on a phosphor, despite this being highly relevant for diagnosing non-neutral plasmas and positron beams used in surface science.

In this letter, for two different phosphors (ZnS:Ag and $\mathrm{ZnO}: \mathrm{Zn}$, which have notably large and notably small dead voltages, respectively), we quantitatively compare the luminescence excited by a positron beam to that excited by an electron beam with a comparable particle flux, for incident energies ranging from $0-5 \mathrm{keV}$. In this regime, penetration depths into solid matter range from less than a single atomic layer [11] up to few hundred nanometers [12], and surface studies with positrons have produced a wealth of new findings through a variety of methods [13-15]. Our results put the widespread use of phosphors for diagnosis in antimatter physics on a sounder footing, while at the same time offering insights into the properties of phosphors and mechanisms of cathodoluminescence.

The luminescent efficiency of electron beams has been studied for a number of materials and beam properties $[6$, 16-18]. Luminescence can be expressed by the product of independent functions of the electron beam energy and beam current, with a linear dependence on current up to current densities far higher than pertains to our work $[6,19]$. We assert that positron response can be described by an expression of the same form and therefore write

$$
\begin{aligned}
L_{ \pm} & =g_{1 \pm}(I) g_{2 \pm}(V) \\
& =I g_{ \pm}(V)
\end{aligned}
$$

where the subscript $+(-)$ refers to positrons (electrons), $L$ is luminescence, and $g_{1}$ and $g_{2}$ are independent functions of the unsigned, single-species beam current $I$ and the attractive phosphor screen bias $V$, respectively. (For electrons, $V=V_{\text {screen }}$, while for positrons, $V=$ $-V_{\text {screen. }}$ ) In Eq. 2, we have used $g_{1}(I)=k I$, and the constant $k$ has been incorporated into $g$. Naively, one would also expect $d g / d V$ to be constant as soon as $e V$ (where $e$ is the elementary charge) exceeds the band gap of the material (e.g., 3.5 or $3.9 \mathrm{eV}$ for $\mathrm{ZnS}$, depending on the polymorph; 3.3 for $\mathrm{ZnO}$ ). For electrons at energies of $10-30 \mathrm{keV}$ (typical of cathode ray tubes), this is indeed the case; an increase in $V$ results in a proportional increase in the number of electron-hole pairs, which in turn generate photons at luminescence centers. At lower voltages, however, it is not; $1-2 \mathrm{kV}$ may need to be applied to produce any detectable luminescence at all, and the transition to linearity may require several more $\mathrm{kV}$. 


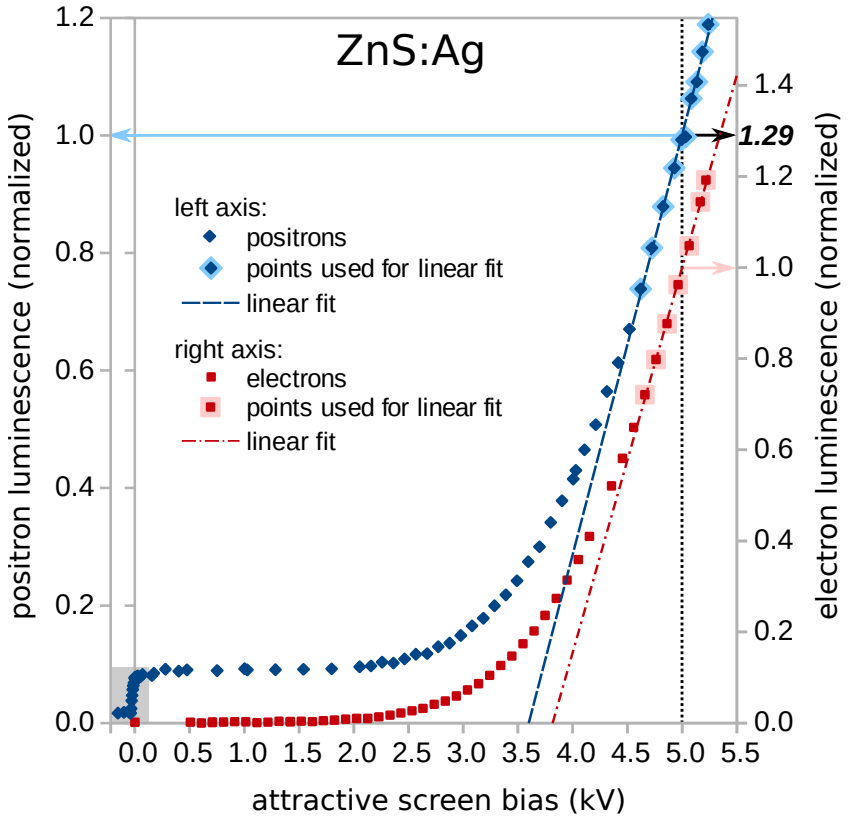

FIG. 1. Dependence of ZnS:Ag luminescence on attractive screen bias for positrons (blue diamonds, left- $y$ axis), and electrons (red squares, right $y$-axis). Each data set is independently normalized to the $5 \mathrm{kV}$ value; uncertainties are on the order of the symbol size or smaller. To facilitate comparisons of absolute luminescence at a glance, the relative scaling of the two vertical axes is chosen to match measurements of luminescence yield; this interspecies comparison, however, has a larger uncertainty. For example, at $V=5 \mathrm{kV}$, positrons produce $L_{+} / L_{-}=1.29 \pm 0.08(6 \%)$ as much luminescence as electrons. The gray box indicates the region shown in greater detail in Fig. 5.

The value of the "dead voltage", the $V$-intercept of the linear part of $g_{-}(V)$, varies with the type of phosphor and its preparation [6]. Electron data exhibiting typical screen bias dependence are shown in Fig. 1. The corresponding positron data plotted alongside illustrate that not only is positron luminescence brighter than electron luminescence, but it also has a distinct functional dependence on incident particle energy.

Experiments were conducted with two different phosphor screens. The first was commercially produced (Kimball Physics) and uses ZnS:Ag Type 1330 (P-22 Blue). The phosphor layer, applied to a conductive glass plate in a stainless steel holder, is $50-70 \mu \mathrm{m}$ thick, corresponding 10-20 layers of $\sim 5-\mu \mathrm{m}$ phosphor particles. The second screen uses an identical plate and holder, to which we applied the phosphor ZnO:Zn (Leuchtstoff GMBH Heidelberg), which is known for its responsiveness to low-energy electrons (implying an unusually low dead voltage), via an ethanol suspension and a standard issue paintbrush. Neither screen had an aluminum layer nor a bonding agent (two frequent additions to phosphor screens), so that incident charged particles interacted directly with the phosphor. Each screen could be biased positively

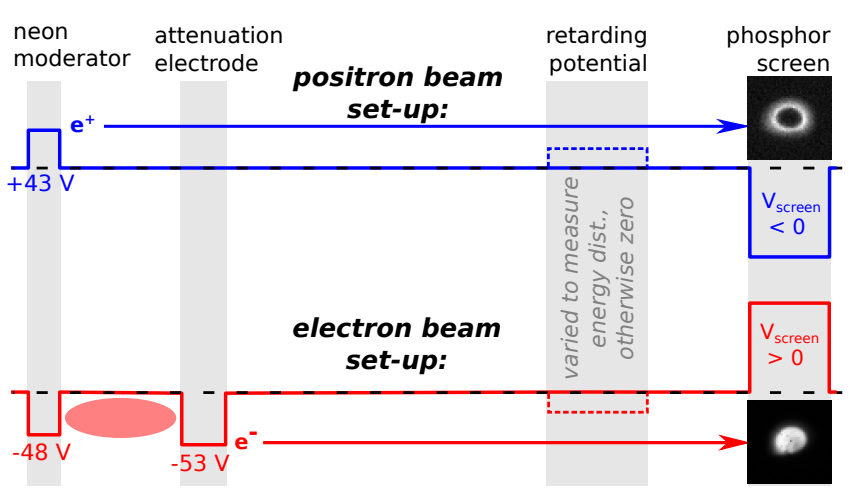

FIG. 2. Illustration of typical electrostatic bias voltages used for positron (top) and electron (bottom) experiments, plus an example image of each beam.

or negatively to about $5 \mathrm{kV}$. Luminescence produced by charged particles was measured by photographing the phosphor layer through the glass plate with a cooled CCD camera (Apogee), subtracting a background image, then summing the pixel counts over a region larger than the beam size. Exposure lengths ranged from 30 to $300 \mathrm{~s}$. Example data can be found in the supplemental information [20].

The positron beam system (First Point Scientific) uses positrons from a ${ }^{22} \mathrm{Na}$ source $(2-4 \mathrm{mCi})$ that are moderated in a thick layer of solid neon; the beam current was $<0.15 \mathrm{pA}$. In order to produce an electron beam for comparison with the positron beam, the moderator was negatively biased to repel secondary electrons produced by the moderation process; the resulting electron beam was then attenuated with a more negative retarding potential to produce beam currents of $0.02-2 \mathrm{pA}$. Each beam was adiabatically magnetically guided $(B=120-800 \mathrm{G})$ around a line-of-sight shield (filtering away unmoderated positrons), then through two sets of cylindrical electrodes en route to the phosphor screen in ultra-high vacuum $\left(10^{-10} \mathrm{mbar}\right)$. Select electrodes could be used to attenuate the beam or to measure the beams' longitudinal energy distributions (Fig. 2); all others were grounded. A typical energy distribution measurement for each species can be found in the supplemental information [21]. The parallel temperature is on the order of some $\mathrm{eV}(2 \mathrm{eV}$ for the electron beam, $6 \mathrm{eV}$ for the positron beam); the perpendicular temperature is expected to be of the same order or less. Thus, the energy spread is small compared to the particles' incident energy for typical ( $\mathrm{kV}$-scale) values of $V$.

As expressed in Eq. 1-2, precise measurements of $I$ are needed to make absolute comparisons between positron and electron luminescence; for the $\sim 0.1-\mathrm{pA}$ positron beam, the current had to be determined to an accuracy of few thousandths of a pA. These were performed with the screen connected to ground through a picoammeter (Keithley). Corresponding luminescence measurements 


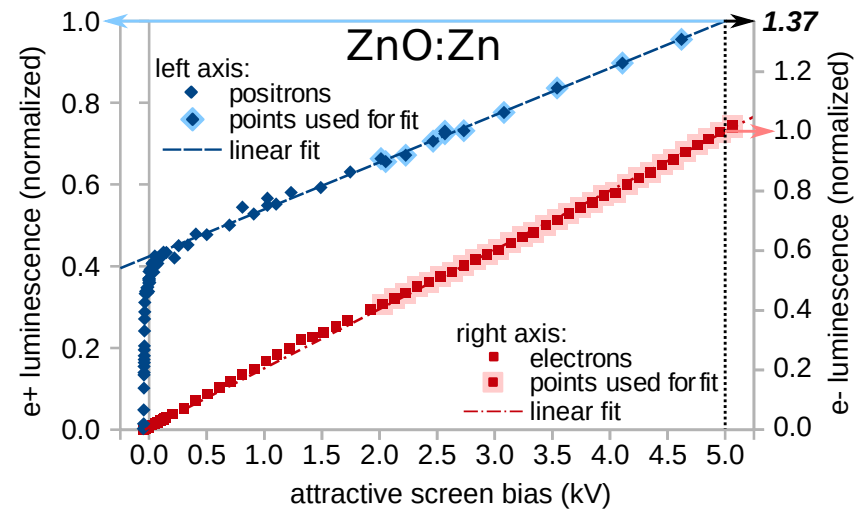

FIG. 3. Dependence of $\mathrm{ZnO}: \mathrm{Zn}$ luminescence on attractive screen bias is plotted for positrons and electrons in the same fashion as in Fig. 1. The relative luminescence yield $L_{+} / L_{-}$ at $V=5 \mathrm{kV}$ is $1.37 \pm 0.15(11 \%)$.

for each beam current were taken at an attractive screen bias of several $\mathrm{kV}$. In all four cases (for both species and both phosphors), this yielded as expected a linear relationship betwen $L$ and $I$ (Eq. 2); additional details and plots can be found in the supplemental information [22]. The assumption that $L$ depends on $V$ and $I$ independently was also observed experimentally. For a given species and phosphor, so long as $e V>>$ the beam energy, varying the phosphor screen bias always produces the same $g(V)=L(V) / I$ curve. This curve is different, however, for each pairing of species and phosphor. Both species' curves are plotted in Fig. 1 for ZnS:Ag and in Fig. 3 for $\mathrm{ZnO}: \mathrm{Zn}$.

For $\mathrm{ZnS}: \mathrm{Ag}$ (Fig. 1), the electron curve $g_{-}(V)$ exhibits the standard non-responsiveness at low voltages and transitions to linear behavior for $V \geq 4.6 \mathrm{kV}$. A fit to points in the linear regime yields a dead voltage of $3.82 \pm 0.02 \mathrm{kV}$ for a $50-\mathrm{eV}$ beam. The positron curve $g_{+}(V)$ also exhibits linear dependence for $V \geq 4.6 \mathrm{kV}$ but has a somewhat lower dead voltage $(3.60 \pm 0.02 \mathrm{kV}$ for a $45-\mathrm{eV}$ beam). Most strikingly, $g_{+}(V)$ does not go to zero as $V \rightarrow 0^{+}$but rather plateaus. The phosphor screen responds to positrons at attractive screen biases well below the threshold for significant electron response, persists even for a positively biased screen, and only disappears when the screen is biased repulsively to within about $10 \mathrm{eV}$ of the beam energy. The small remaining luminescence beyond the cut-off $(V<-40 \mathrm{~V})$ constitutes a little less than $2 \%$ of the total luminescence at $V=5$ $\mathrm{keV}$ and can be attributed to a small population of highenergy positrons $(>150 \mathrm{eV})$ also seen in the beam energy distribution measurement [23].

For $\mathrm{ZnO}: \mathrm{Zn}$ (Fig. 3), no dead voltage is identifiable for either species; both positrons and electrons produce luminescence as soon as they have enough kinetic energy to reach the screen - i.e., the $V$ threshold for detection of luminescence from a repulsively biased screen is located precisely at the beam energy. The $g_{-}(V)$ and $g_{+}(V)$ curves are nevertheless highly distinct for this phosphor as well. Whereas $g_{-}(V)$ is almost perfectly linear over the entire range of our measurements, $g_{+}(V)$ initially rises orders of magnitude faster, with the same width in $V$ as the beam's energy distribution measurement. In other words, as soon as positrons can reach the screen electrostatically, they produce as much luminescence as electrons with $\sim 2.5 \mathrm{keV}$ of kinetic energy.

Comparisons such as this (of the absolute luminescence of one species to the other) follow from the current calibration - i.e., how many image counts are generated per $\mathrm{pA}$ of positrons or electrons. Results indicate that for the same $I, 5-\mathrm{keV}$ positrons produce more luminescence than $5-\mathrm{keV}$ electrons by a factor of $\left.\left(L_{+} / L_{-}\right)\right|_{5 \mathrm{kV}}=g_{+}(5$ $\mathrm{kV}) / g_{-}(5 \mathrm{kV})=1.29 \pm 0.08$ for $\mathrm{ZnS}: \mathrm{Ag}$ and $1.37 \pm 0.15$ for $\mathrm{ZnO}: \mathrm{Zn}$. This information was used to set the relative scaling of the left and right y-axes in Figs. 1 and 3. Clearly, both the electron luminescence fraction $L_{-} / L_{+}$ and the excess positron luminescence $L_{+}-L_{-}$depend on particles' incident kinetic energy. These values and their uncertainty have been calculated for both phosphors for the entire energy range of the experiment, and plots are provided in the supplemental information [24]. Of particular note is the monotonic increase of the electron luminescence fraction; in the limit of high incident particle energies, the difference between positron-induced and electron-induced luminescence is expected to become negligible compared to the total amount of luminescence produced by either species, consistent with the two previous quantitative comparisons of positron and electron luminescence that we found in the literature, both done with higher-energy beams $(\geq 30-\mathrm{keV})[8,9]$.

It is clear that kinetic energy is not the only means by which positrons produce luminescence; they also do so through annihilation with electrons. Annihilation produces at least one hole per positron; it can also initiate the Auger process (in which a second electron from a higher energy level falls into the hole produced by annihilation with the first, and the difference in energy between the two levels is imparted to a third electron), resulting in two holes and one excited electron [25-28] (or more, in the event of an Auger cascade [29-32]). Annihilation with core electrons is relatively unlikely; when it does occur, an excited electron can have a kinetic energy significantly larger than the original incident positron and thereby excite additional electron/hole pairs through collisions, but this is still only in the tens or hundreds of $\mathrm{eV}$ $[26,33]$. (Thus, this particular pathway is not expected to contribute significantly to the large effect we observe, in which positrons with a few $\mathrm{eV}$ or tens of $\mathrm{eV}$ of energy produce as much luminescence as $\mathrm{keV}$-scale electrons.) Annihilation with valence electrons is much more probable, and recent studies in graphene found that $80-100 \%$ of deep valence-band holes were filled via an Auger transition [28]. When the valence band is wide enough (e.g., 

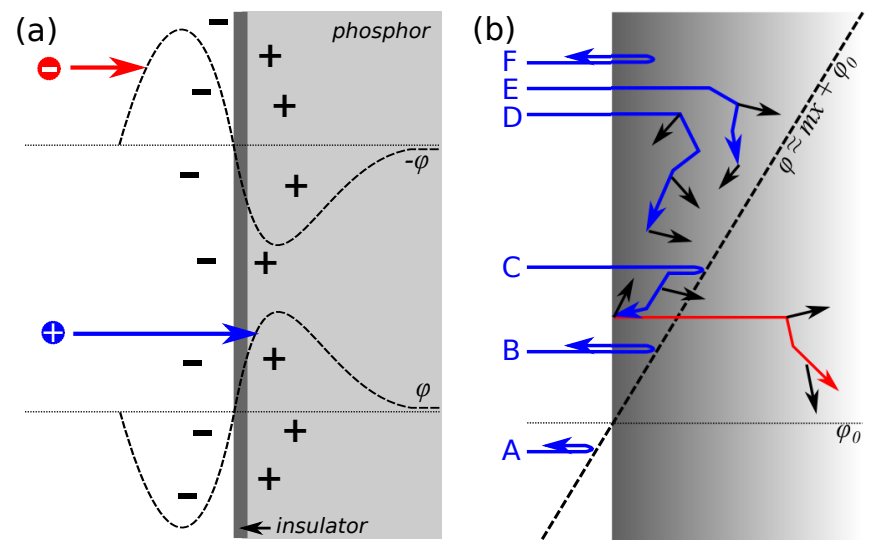

FIG. 4. a.) Illustration of a surface-bound electron layer, stabilized by a sub-surface population of holes. The resulting electrostatic potential $\phi$ (dashed line) prevents low-energy electrons (but not positrons) from reaching the surface of the material. b.) There are many different mechanisms by which positrons interact with the phosphor surface (gray) and/or, if present, a sub-surface electrostatic potential (dashed line). These include electrostatic reflection (trajectories A,B,C), inelastic collisions $(\mathrm{C}, \mathrm{D}, \mathrm{E})$, annihilation with a core electron (C), annihilation with valence electrons $(\mathrm{D}, \mathrm{E})$, and backscattering $(\mathrm{F})$. Blue arrows represent positrons, the red arrow represents an electron excited by Auger processes following core annihilation, and black arrows represent both conductionband electrons and valence-band holes, which then lead to luminescence.

$20 \mathrm{eV}$ in graphene), electrons excited by these "VVV transitions" have sufficient energy to overcome the work function, escape the material, and be observed in the positron annihilation-induced Auger electron spectrum (PAES); in materials with narrower valence bands, VVV transitions may instead excite electrons merely into the conduction band, where they cannot be detected with PAES. In luminescent materials, however, conductionband electrons can then travel to luminescence centers and there combine with valence-band holes to produce photons. Additionally, if luminescence centers involve defects that act as positron traps - e.g., certain types of vacancies $[3,5,15]$ - positrons will preferentially annihilate there, creating holes directly at those locations in the crystal structure. Thus, there is significant potential for positron luminescence to provide new information about open questions regarding luminescent materials, which may include the structure of luminescence centers $[3,4]$, the excitation and relaxation pathways $[1,5]$, and/or the origin of the dead voltage $[2,6]$.

Several mechanisms for the dead voltage have been proposed over the decades. One explanation is a nonluminescent surface layer (formed during the phosphor production process), through which electrons must penetrate in order to reach the phosphor and excite electronhole pairs [17]. A second explanation is an enhanced propensity for non-radiative recombination of electrons

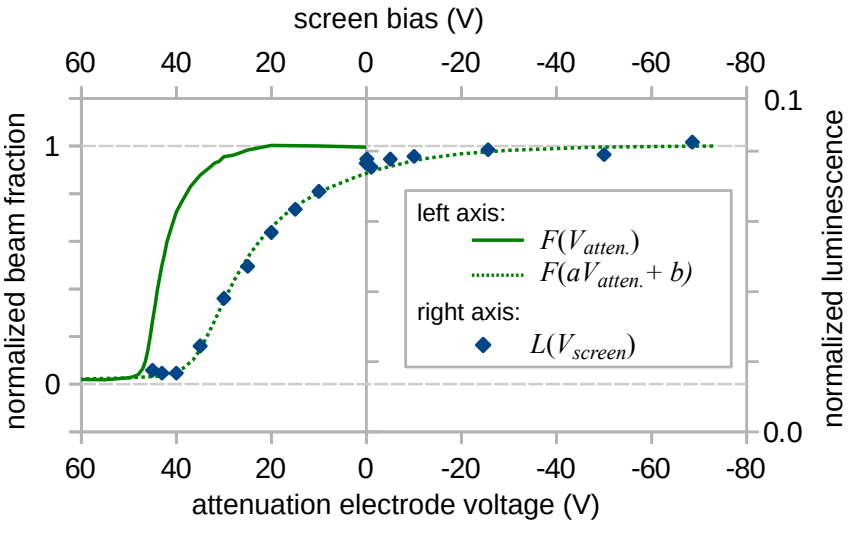

FIG. 5. The ZnS:Ag positron luminescence cut-off is plotted on the right $y$-axis as a function of screen bias (diamond symbols); uncertainties are on the order of the symbol size. Plotted on the left $y$-axis are the cumulative energy distribution of the beam (solid green line), and the transformation of this curve (dotted green line), as discussed in the text, that provides the best match to the cut-off $(a=3, b=-103)$. Horizontal dashed lines indicate normalized beam fractions of $0 \%$ and $100 \%$.

and holes near the surface due to surface states and/or band-bending [2]. A third explanation proposes that if a phosphor particle is coated with any insulating contaminant, however thin, a "surface bound electron layer" (Fig. 4a) will form, extending several $\mu \mathrm{m}$ into the vacuum from the surface; stabilized by a layer of sub-surface holes, this creates a region of negative electrostatic potential, akin to those seen on insulators in scanning electron microscopes (SEMs) [34], that incident electrons must have enough energy to penetrate $[1,6]$. Because positrons and electrons are oppositely charged but have similar characteristics with respect to penetration into solids $[12,13]$ and secondary electron production [35], comparing $g_{+}(V)$ to $g_{-}(V)$ can help to discriminate among these possibilities.

That positrons in $\mathrm{ZnS}: \mathrm{Ag}$ produce significant luminescence well below the dead voltage is consistent with the third model, as the others would be expected to inhibit positron-induced luminescence in a similar fashion as electron-induced luminescence. (Note that there are not known to be Auger processes capable of producing electrons with energies above the dead voltage with nonvanishing probabilities [26].) Whereas the negative electrostatic potential of the surface bound electron layer prevents low-energy electrons from accessing the phosphor surface entirely, low-energy positrons can reach the surface and then undergo various elastic and inelastic processes (Fig. 4b). Furthermore, Fig. 5 shows that the relationship between the $\mathrm{ZnS}: \mathrm{Ag}$ positron luminescence cut-off and the positron beam's cumulative energy distribution $F\left(V_{\text {atten }}\right)$ is consistent with the presence of a sub-surface electrostatic potential $\phi$ that increases linearly with depth (Fig. 4b). In this picture, the likelihood 
that positrons with just enough kinetic energy to access the surface (B, C) will undergo inelastic scattering ultimately leading to luminescence - is proportional to path length in the material. Path length (restricted by $\phi)$ rises linearly with incident energy. As a result, instead of occurring at the beam energy - as one would expect and as is observed for $\mathrm{ZnO}: \mathrm{Zn}$ - the $L(V)$ cut-off is offset and stretched by constants relating to the potential at the surface and the length scale over which it rises.

In summary, we have performed quantitative comparisons of the luminescence produced by 0 - to $5-\mathrm{keV}$ positron and electron beams incident on phosphors and found that positrons produce more brightness than electrons over the entire energy range. Of particular note is that positrons with an incident energy of just a few $\mathrm{eV}$ (in the case of $\mathrm{ZnO}: \mathrm{Zn}$ ) or a few tens of $\mathrm{eV}$ (in the case of $\mathrm{ZnS}: \mathrm{Ag}$ ) produce as much luminescence as 2.5to $3.5-\mathrm{keV}$ electrons. These findings are expected to have significant utility for non-perturbatively diagnosing low-energy positron beams and plasmas (relevant to electron/positron pair plasma creation, anti-hydrogen experiments, slow positron scattering processes, and positronium ionization studies, among other applications). They also represent compelling evidence for the excitation of luminescence via annihiliation and Auger processes, as well as a new way of addressing decades-old questions in cathodoluminescence.

This work has been funded by the Helmholtz Association Postdoc Programme. The authors would also like to thank the Surko Group (University of California, San Diego) for the loan of their positron source; M. Otte for contributing a sample of $\mathrm{ZnO}: \mathrm{Zn} ;$ J. R. Danielson for the tip about producing an electron beam from the moderator; B. L. Standley, J. Horn-Stanja, and C. Richter for valuable discussions; and X. Sarasola and N. Paschkowski for their assistance in assembling the experiment.

* evs@ipp.mpg.de

[1] L. Ozawa and M. Itoh, Chemical Reviews 103, 3835 (2003), http://dx.doi.org/10.1021/cr0203490.

[2] B. L. Abrams and P. H. Holloway, Chemical Reviews 104, 5783 (2004), http://dx.doi.org/10.1021/cr020351r.

[3] J. Ji, A. M. Colosimo, W. Anwand, L. A. Boatner, A. Wagner, P. S. Stepanov, T. T. Trinh, M. O. Liedke, R. Krause-Rehberg, T. E. Cowan, and F. A. Selim, Scientific Reports 6, 31238 (2016).

[4] P. A. Rodnyi and I. V. Khodyuk, Optics and Spectroscopy 111, 776 (2011).

[5] C. S. Kang, P. Beverley, P. Phipps, and R. H. Bube, Phys. Rev. 156, 998 (1967).

[6] M. Itoh and L. Ozawa, Annu. Rep. Prog. Chem., Sect. C: Phys. Chem. 102, 12 (2006).

[7] J. R. Danielson, D. H. E. Dubin, R. G. Greaves, and C. M. Surko, Rev. Mod. Phys. 87, 247 (2015).

[8] K. Gubernator, P. Heckmann, and A. Flammersfeld,
Zeitschrift fuer Physik 158, 268 (1960).

[9] O. I. Meshkov, V. V. Smaluk, D. P. Sukhanov, E. N. Galashov, V. Dorokhov, A. N. Zhuravlev, and V. A. Kiselev, Proceedings of the 10th European Workshop on Beam Diagnostics and Instrumentation for Particle Accelerators, 558 (2011).

[10] J. Xu, L. D. J. Hulett, T. A. Lewis, and N. H. Tolk, Studies of positron induced luminescence from polymers, Tech. Rep. (United States, 1994) 1994; 5 p; 10. international conference on positron annihilation; Beijing (China); 23-29 May 1994; CONTRACT AC05-84OR21400; Also available from OSTI as DE94013622; NTIS; US Govt. Printing Office Dep.

[11] S. Mukherjee, M. P. Nadesalingam, P. Guagliardo, A. D. Sergeant, B. Barbiellini, J. F. Williams, N. G. Fazleev, and A. H. Weiss, Phys. Rev. Lett. 104, 247403 (2010).

[12] S. Valkealahti and R. M. Nieminen, Applied Physics A 32, 95 (1983).

[13] P. J. Schultz and K. G. Lynn, Reviews of Modern Physics 60, 701 (1988).

[14] M. J. Puska and R. M. Nieminen, Rev. Mod. Phys. 66, 841 (1994).

[15] C. Hugenschmidt, Surface Science Reports 71, 547 (2016).

[16] S. T. Martin and L. B. Headrick, Journal of Applied Physics 10, 116 (1939).

[17] J. Kingsley and J. Prener, Journal of Applied Physics 43, 3073 (1972).

[18] D. E. Gutzler, Journal of The Electrochemical Society 126, $571 \quad$ (1979), http://jes.ecsdl.org/content/126/4/571.full.pdf+html.

[19] S. Myhajlenko, in Luminescence of Solids, edited by D. R. Vij (Plenum Press, New York, 1998).

[20] See supplemental information.

[21] See supplemental information.

[22] See supplemental information.

[23] See supplemental information.

[24] See supplemental information.

[25] A. Weiss, R. Mayer, M. Jibaly, C. Lei, D. Mehl, and K. G. Lynn, Phys. Rev. Lett. 61, 2245 (1988).

[26] K. O. Jensen and A. Weiss, Phys. Rev. B 41, 3928 (1990).

[27] E. Jung, H. Zhou, J. Kim, S. Starnes, R. Venkataraman, and A. Weiss, Applied Surface Science 116, 318 (1997), proceedings of the Seventh International Workshop on Slow-Positron Beam Techniques for Solids and Surfaces.

[28] V. A. Chirayath, V. Callewaert, A. J. Fairchild, M. D. Chrysler, R. W. Gladen, A. D. Mcdonald, S. K. Imam, K. Shastry, A. R. Koymen, R. Saniz, B. Barbiellini, K. Rajeshwar, B. Partoens, and A. H. Weiss, Nature Communications 8, 16116 (2017).

[29] M. Wolfsberg and M. L. Perlman, Phys. Rev. 99, 1833 (1955).

[30] L. Partanen, Auger cascade processes in xenon and krypton studied by electron and ion spectroscopy, Ph.D. thesis, University of Oulu, Finland (2007).

[31] V. Stumpf, K. Gokhberg, and L. S. Cederbaum, Nat. Chem. 8, 237241 (2016).

[32] D. You, H. Fukuzawa, Y. Sakakibara, T. Takanashi, Y. Ito, G. G. Maliyar, K. Motomura, K. Nagaya, T. Nishiyama, K. Asa, Y. Sato, N. Saito, M. Oura, M. Schffler, G. Kastirke, U. Hergenhahn, V. Stumpf, K. Gokhberg, A. I. Kuleff, L. S. Cederbaum, and K. Ueda, Nature Communications 8, 14277 (2017).

[33] P. Palmberg and P. E. Industries, Handbook of Auger 
Electron Spectroscopy: A Reference Book of Standard Data for Identification and Interpretation of Auger Electron Spectroscopy Data (Physical Electronics Industries, 1972).

[34] M. Belhaj, O. Jbara, M. Filippov, E. Rau, and M. An- drianov, Applied Surface Science 177, 58 (2001).

[35] E. Jung, R. Venkataraman, S. Starnes, and A. H. Weiss, in Positron Annihilation - ICPA-11, Materials Science Forum, Vol. 255 (Trans Tech Publications, 1997) pp. 708 710 . 